\title{
A brief look at model-based dose calculation principles, practicalities, and promise
}

\author{
Ron S. Sloboda, PhD, FCCPM!', Hali Morrison, PhD candidate,2, Brie Cawston-Grant, MSc',2, \\ Geetha V. Menon, PhD, FCCPM!'2 \\ 'Department of Oncology, Faculty of Medicine and Dentistry, University of Alberta, Edmonton, ${ }^{2}$ Department of Medical Physics, \\ Cross Cancer Institute, Alberta Health Services, Edmonton, Alberta, Canada
}

\begin{abstract}
Model-based dose calculation algorithms (MBDCAs) have recently emerged as potential successors to the highly practical, but sometimes inaccurate TG- 43 formalism for brachytherapy treatment planning. So named for their capacity to more accurately calculate dose deposition in a patient using information from medical images, these approaches to solve the linear Boltzmann radiation transport equation include point kernel superposition, the discrete ordinates method, and Monte Carlo simulation. In this overview, we describe three MBDCAs that are commercially available at the present time, and identify guidance from professional societies and the broader peer-reviewed literature intended to facilitate their safe and appropriate use. We also highlight several important considerations to keep in mind when introducing an MBDCA into clinical practice, and look briefly at early applications reported in the literature and selected from our own ongoing work. The enhanced dose calculation accuracy offered by a MBDCA comes at the additional cost of modelling the geometry and material composition of the patient in treatment position (as determined from imaging), and the treatment applicator (as characterized by the vendor). The adequacy of these inputs and of the radiation source model, which needs to be assessed for each treatment site, treatment technique, and radiation source type, determines the accuracy of the resultant dose calculations. Although new challenges associated with their familiarization, commissioning, clinical implementation, and quality assurance exist, MBDCAs clearly afford an opportunity to improve brachytherapy practice, particularly for low-energy sources.

J Contemp Brachytherapy 2017; 9, 1: 79-88 DOI: https://doi.org/10.5114/jcb.2017.65849
\end{abstract}

Key words: dose calculation, MBDCA, Monte Carlo, TG-43.

\section{Purpose}

Since its introduction more than twenty years ago, the American Association of Physicists in Medicine Task Group 43 (AAPM TG-43) dose calculation formalism for photon-emitting radionuclide sources $[1,2]$ has become a mainstay of brachytherapy dosimetry clinical practice. Originally developed for low-energy interstitial implants, the formalism has subsequently been applied broadly to intracavitary and superficial treatments [3], and to treatments delivered using miniaturized electronic radiation sources [4], so that today it is recognized as a de facto international standard. TG-43 dose calculation methodology is based on the parameterization and superposition of single-source dose distributions, obtained in liquid water under fixed geometry conditions approximating full scatter, and incorporates the assumption that these distributions have cylindrical symmetry.

Demonstrable benefits to the brachytherapy community accruing from the use of the TG-43 formalism include standardization of the dose calculation method and of the single-source dosimetry parameter sets, and simplicity and universality of implementation in commercial treatment planning systems (TPSs). Such extensive standardization has brought a high level of consistency to the comparison of treatment regimens and clinical outcomes across institutions, a feature that is especially valued in clinical trials research. Nevertheless, despite these benefits, the TG-43 approach is limited by several inherent simplifications as noted by Rivard et al. [5]. These are based in the radiological physics domain and involve a lack of consideration of: 1) differences between absorbed dose in water and tissues; 2) differences between radiation attenuation in water and tissues; 3 ) radiation interactions with an applicator or other sources; 4) radiation scattering conditions in and immediately adjacent to the patient, and 5) dose contributions from electrons. The extent to which each limitation influences the accuracy of dose calculation depends upon the anatomic site and the energy of the radiation source. For example, in ${ }^{192}$ Ir breast treatments, the lack of consideration of scattering conditions near the
Address for correspondence: Ron S. Sloboda, PhD, FCCPM, Department of Oncology, Faculty of Medicine and Dentistry, University of Alberta, Edmonton, Cross Cancer Institute, Room 0418, 11560 University Avenue, Edmonton, Alberta, Canada T6G 1Z2, phone: +1 7804328719 ,

fax: +1 780432 8615, e-mail: ron.sloboda@ahs.ca
Received: 26.09 .2016

Accepted: 07.01.2017

Published: 28.02.2017 
skin surface can lead to skin dose estimates that are more than 5\% higher [5]. For another example, in ${ }^{103} \mathrm{Pd}$ and ${ }^{125} \mathrm{I}$ prostate implants, inter-seed attenuation can potentially reduce dose by more than $10 \%$ along the needle insertion direction and lower planning target volume (PTV) $\mathrm{D}_{90}$ by as much as 5\% [6]. Rivard et al. [5] conveniently summarized the sensitivity of the most common anatomic sites to the aforementioned dosimetric limitations in tabular form, and observed that most applications of brachytherapy are impacted to some degree.

As 3D image-based, volume-optimized treatment planning began to replace film-based planning in brachytherapy, more sophisticated approaches to dose calculation having their origins in nuclear science and the nuclear industry were adapted to meet the need for greater dose calculation accuracy. Collectively referred to as model-based dose calculation algorithms (MBDCAs) by AAPM TG-186 [7] for their capacity to calculate dose deposition in a patient using information from medical images, these algorithms employ a variety of methods to improve calculation accuracy. The resulting improvements are expected to be greatest for low-energy $(<50 \mathrm{keV})$ sources, for which dose deposition is demonstrably most sensitive to differences in tissue and applicator composition. As a result of concerted developmental efforts, motivated by perceived clinical need, a number of different algorithms have found their way into commercial TPSs.

In this article, we briefly describe the commercial MBDCAs that are currently available, identify professional society guidance intended to facilitate their safe and appropriate use, and highlight some considerations associated with their introduction into clinical practice. We also look at early applications of these MBDCAs including some examples from our own work in progress, and conclude with a summary of their current and anticipated future benefits. Given the article's broad scope, the approach taken has been to present material concisely and to provide salient references for readers desiring more detail.

\section{Commercial treatment planning systems incorporating model-based dose calculation algorithms}

\section{Model-based dose calculation algorithm implementations}

Presently, commercial treatment planning systems that incorporate a MBDCA include BrachyVision (Varian Medical Systems, Palo Alto, CA) for ${ }^{192}$ Ir high-doserate (HDR) and pulsed-dose-rate (PDR) brachytherapy, Oncentra Brachy for ${ }^{192} \mathrm{Ir}$ HDR, ${ }^{192} \mathrm{Ir}$ PDR, and ${ }^{60} \mathrm{Co}$ HDR brachytherapy (Nucletron, an Elekta company, Elekta AB, Stockholm, Sweden), as well as Radiance (GMV SA, Madrid, ES) for intraoperative radiotherapy with low-energy X-rays $(<50 \mathrm{keV})$, which in several respects is similar to electronic brachytherapy. The following brief overview and comparison of the three MBDCAs available in these TPSs were gleaned from product-related literature $[8,9,10]$, white papers [11,12], and a recent review of brachytherapy dose calculation algorithms [13].
In terms of radiation physics, the transport of particles (photons, electrons, protons, etc.) through matter and their interaction with it is governed by the linear Boltzmann transport equation (LBTE). For any given irradiation set-up, involving an inhomogeneous medium, solutions can be obtained using one of three main approaches: 1) Monte Carlo (MC) simulation, which tracks a large number of particles undergoing successive random interactions in the medium; 2) grid-based numerical methods, which directly solve a discretized version of the equation, and 3) point kernel superposition methods, which take a solution for a much simpler set-up and adapt it to the setup at hand. The MBDCAs available in the three commercial TPSs identified above each take a unique approach to solving the LBTE for photon-emitting brachytherapy sources, but with some notable similarities. These include performing dose calculations on a variably-sized 3D Cartesian grid for efficiency, assuming charged-particle equilibrium so that dose can be equated to collisional kerma, employing a primary/scatter dose separation framework [14] enabling the dose from primary photons (photons exiting the source not scattered in the medium) to be calculated analytically, and reporting the dose to local medium for transport in full medium, $D_{\mathrm{m}, \mathrm{m}^{\prime}}$ as defined and recommended by AAPM TG-186 [7]. As well, two of the MBDCAs (AcurosBV in BrachyVision and the Advanced Collapsed-cone Engine [ACE] in Oncentra Brachy) use a semi-analytic first-scatter distributed source approach to mitigate ray-effect artifacts that can arise from photon angle discretization [15].

First released in 2009, the AcurosBV MBDCA in BrachyVision was inspired by Attila [15] (Transpire Inc., Gig Harbor WA, USA), a general-purpose software system for radiation transport calculations that originated in the Los Alamos National Laboratory. AcurosBV is an independent, dedicated software package that employs a numerical solution method optimized specifically for brachytherapy dose calculations. Supported radiation sources are individually modelled as one or more point sources representing the energy and angular dependent photon intensity emitted from the source surface. AcurosBV solves the LBTE directly by discretizing all six of its independent variables: space (3 variables), photon angle ( 2 variables), and photon energy ( 1 variable). The fineness or coarseness of the discretizations affects both the accuracy and speed of dose calculation, and so an appropriate balance is required. This balance has been optimized for ${ }^{192}$ Ir sources in the current software, and cannot be modified by the user. A potential issue with angular discretization methods is the presence of 'ray-line' artifacts in the dose distribution, however these have been essentially eliminated in AcurosBV [16]. Early versions of the software reported only dose to water for photon transport in full medium, $D_{\mathrm{w}, \mathrm{m}}$; however, since 2013, the user has been offered a choice between $D_{\mathrm{m}, \mathrm{m}}$ and $D_{\mathrm{w}, \mathrm{m}}$ [8]. Studies indicate there is little difference between $D_{\mathrm{m}, \mathrm{m}}$ and $D_{\mathrm{w}, \mathrm{m}}$ in soft tissues for ${ }^{192} \mathrm{Ir}$ sources, but potentially large differences for low-energy sources such as ${ }^{125} \mathrm{I}[7,17,18,19]$. A complete description of AcurosBV and its approach to solving the LBTE for brachytherapy applications can be found in Varian Brachytherapy product literature [8]. 
The ACE MBDCA in Oncentra Brachy, first released in 2014, is the result of research done to extend the collapsed-cone convolution/superposition (CCCS) dose calculation method used in external beam radiotherapy planning to brachytherapy. Supported radiation sources are individually modelled as anisotropic point emitters, with near-source corrections (obtained from MC simulation) applied for the finite extent of the source, for dose gradient averaging over finite voxels, and to a limited extent, for electron transport. Advanced Collapsed-cone Engine calculates the dose as the sum of three components representing contributions from primary, once-scattered, and multiply-scattered photons [11]. In principle, the first two components solve the LBTE, whereas the last component is an approximation. Once- and multiply-scattered dose contributions are successively calculated by CCC of the local photon fluence with pre-computed point dose kernels. The dose kernels are generated in a fixed-size water volume using MC simulation and scaled to reflect the influence of each different material in the inhomogeneous medium. Both the fixed water volume size and scaling operations are approximations that can limit algorithm performance in certain circumstances [20,21]. Parameters in the calculation that influence accuracy and over which the user is given limited control include the numbers of photon transport directions (coincident with the collapsed cone axes) for once- and multiply-scattered photons. As for AcurosBV, angular discretization (in the form of photon transport directions) brings with it the possibility of 'ray-line' artifacts, which for ACE are comparatively more pronounced, but of limited clinical concern [16]. A comprehensive description of the implementation can be found in a recent publication dedicated to the topic [9].

Finally, the hybrid MC algorithm in the Radiance TPS, initially released in 2015, was developed specifically for intra-operative radiotherapy. It combines ray-tracing to obtain primary dose with a custom MC code to obtain scatter dose for a photon beam generated by a miniaturized linear accelerator (XRS 4), and collimated by an applicator. The algorithm is made to execute quickly by considering only photoelectric interactions and the first two orders of Compton scattering, incorporating a condensed history method for photon transport, and employing variance reduction techniques. As each XRS 4/applicator combination typically has a different photon emission spectrum, the algorithm does its work in two phases. In an initial, commissioning phase, it models the emission spectrum by simulating radiation transport in the applicator and then weighting the spectrum to fit a measured percentage depth dose curve in water medium [22]. The result is a phase space file that is used in the ensuing clinical application phase, as the source of primary photons for radiation transport simulation in the treatment medium and dose calculation in a user-defined volume of interest. The approach is more fully described in a conference abstract [23], a white paper [12], and an appendix in the Radiance User's Manual [10]. However, at this time, detailed information regarding algorithm implementation is limited.

\section{Model-based dose calculation algorithm inputs}

In contrast to the TG-43 formalism, which is based on the simplifying (and convenient) assumptions identified earlier, the model-based approach to dose calculation requires that the treatment applicator and the patient anatomy in the vicinity of the treatment site be adequately described, in addition to the radiation source. This involves specifying photon emissions from the radiation source, and geometry and material compositions for the treatment applicator and patient. Vendors have integrated the first two inputs into the TPS in the form of predefined source models and applicator libraries, respectively. The user selects a source and applicator model, and then places and orients the applicator within a virtual representation of the patient obtained from 3D images, most commonly CT scans. The 3D image set forms the basis for the third input, the patient anatomy description, which must be constructed by the user. This is done by delineating structures of interest through the familiar process of contouring, and then assigning the material properties of chemical composition and mass density to each of them. The assignment can be done in several ways: 1 ) by selecting from a predefined list, a material whose properties are uniformly assigned to all voxels in a structure; 2) for CT images, by stipulating that a mapping of Hounsfield numbers to materials be used, which assigns material properties to each voxel individually in a structure; 3 ) for CT images, by assigning chemical composition via 1 . and mass density via 2 . TG-186 recommends method 3 . over method 2., as some of the mappings in 2 . cannot be done uniquely.

All MBDCA implementations involve a tradeoff between dose calculation accuracy and execution speed, over which a TPS might provide the user some control. For example, Oncentra Brachy does this for the ACE algorithm by offering a choice between standard (SACE) and high accuracy (hACE) calculation, the latter involving a greater number of transport directions and so requiring a longer execution time. Such a choice, if available, needs to be made on an individual treatment basis after gaining experience with a MBDCA, as described in the next section.

\section{Model-based dose calculation algorithm commissioning and use}

Commissioning a brachytherapy TPS involves verifying the proper functioning of key system components and of the overall system. General guidance in carrying out these tasks can be found in professional society reports such as AAPM TG-40 [24], AAPM TG-56 [25], ESTRO booklet 8 [26], and specifically for HDR ${ }^{192} \mathrm{Ir}$ systems, AAPM TG-59 [27]. When the TPS incorporates a MBDCA, additional algorithm-specific tasks need to be done as recommended in the AAPM/ESTRO/ABG TG-186 report [7]. Recently, a web-based MBDCA commissioning Registry was opened to support physicists and dosimetrists in performing these additional tasks, as reported at the 2016 World Congress of Brachytherapy [28] by the joint AAPM/ESTRO/ABG working group on 
MBDCAs in brachytherapy [16,29]. In brief, the Registry enables an MBDCA user to compare a locally calculated dose distribution with a reference dose distribution generated, using MC simulation, for a series of well-defined 'test plans'. Commissioning resources available on the Registry are presently limited to an initial set of four generic test plans for HDR ${ }^{192}$ Ir brachytherapy; however, these will be augmented with clinical site-specific HDR ${ }^{192}$ Ir test plans and eventually extended to include ${ }^{125} \mathrm{I}$ brachytherapy. The peer-reviewed literature also offers guidance and resources to aid in MBDCA commissioning $[30,31,32,33,34]$.

The introduction of a MBDCA in a clinical setting requires careful preparation. The dose distribution can differ substantially from that obtained using TG-43 [7], and therefore, it is essential to assess how such differences might impact clinical practice. This needs to be done on a treatment site- and technique-specific basis, in order to ascertain whether current prescription doses and dose limits on organs at risk require revision for MBDCA dosimetry. For this reason, professional societies recommend that treatment providers perform TG- 43 and MBDCA dosimetry in parallel and carefully compare results for a period of time sufficient to gain confidence in making the clinical transition to a MBDCA $[7,35,36]$. Some clinically-motivated examples illustrating this parallel dosimetry approach can be found in the recent review article by Papagiannis et al. [13]. It should also be noted that because the commercial MBDCAs described above each use a different dose calculation method whose implementation is proprietary and might change over time, performing parallel dosimetry is also advisable when transitioning from one TPS to another.

A general expectation is that the use of a MBDCA will provide more accurate dose calculations for brachytherapy treatment planning. However, as perceptively noted by Mikell et al., this premise rests on a number of key assumptions [37] that are important to bear in mind and that require careful scrutiny:

1) radioactive sources are sufficiently well modeled;

2) applicator models in TPS libraries are accurate;

3) CT/MRI images are properly converted to materials and material densities;

4) radiation transport sufficiently approximates a solution to the linear Boltzmann transport equation;

5) spatial and temporal aspects of the treatment plan (applicator position, source dwell positions, dwell times, and anatomy) match those of the treatment delivery.

The literature contains several instructive examples of the type of work required to test the preceding assumptions. A single example for each involving a HDR ${ }^{192} \mathrm{Ir}$ source is cited for the benefit of interested readers:

1) Figure 1 in Ma et al. [31] presents dose ratio maps for ACE/TG-43 calculations in water medium (TG-186 level 1 commissioning);

2) Mikell et al. [37] describe verification of a shielded applicator model for cervix brachytherapy;

3) Mikell et al. [38] report that rectal dose parameters in cervix brachytherapy may be sensitive to the mapping of radiopaque packing material;
4) Zourari et al. [39] note that for an esophagus treatment plan created using a voxelized thorax phantom, AcurosBV doses differ from MC by up to $6 \%$ at points lying close to directions defined by primary photon ray-paths tangential to cylindrical trachea and spine structures;

5) Ma et al. [31] caution within the context of a breast case that a sufficiently large CT/MR image volume be scanned and imported into the TPS to enable the MBDCA to properly calculate the scatter contribution to dose, which is especially important in low-dose regions containing organs at risk (OARs).

Finally, it should be reiterated that AcurosBV includes the option to calculate $D_{\mathrm{w}, \mathrm{m}}$ rather than $D_{\mathrm{m}, \mathrm{m}}$ [8]. Users need to be aware of this option and should choose $D_{\mathrm{m}, \mathrm{m}}$ for clinically related work with ${ }^{192} \mathrm{Ir}$ sources as recommended by TG-186 [7], unless they are conducting an investigation that requires $D_{\mathrm{w}, \mathrm{m}}$.

\section{Experience using commercial model-based dose calculation algorithms}

Clinically relevant experience using commercial MBDCAs is limited as these algorithms became available only recently. In the following sections, we present selected accounts from the literature and from some of our own work in progress.

\section{Literature reports}

\section{Cervix}

The earliest work with MBDCAs focused on cervix treatments delivered using unshielded tandem and ovoids applicators. Mikell et al. conducted a retrospective assessment of the impact of heterogeneities on CT-based, conventional Manchester system HDR ${ }^{192}$ Ir treatment plans for 26 patients using AcurosBV in BrachyVision v.8.8 software [38]. Reporting $D_{\mathrm{w}, \mathrm{m}}$, they found minor changes in Point $A$ and $B$ doses, and in $D_{2 c c}$ for rectum, bladder, and sigmoid, with all dose parameters for individual patients differing from TG-43 values by $<5 \%$. Larger differences of up to $\sim 10 \%$ observed at locations within the imaging volume were shown to be attributable to three factors: radiation source modelling and patient boundary, applicator modelling, and tissue heterogeneity. A similar retrospective study also reporting $D_{\mathrm{w}, \mathrm{m}}$ by Hyer et al. comparing eight HDR ${ }^{192}$ Ir plans created with BrachyVision v.8.9 using both conventional and volume-optimization methods reported that heterogeneity corrections had only a small impact on TG-43 calculated dosimetric parameters [40]. Cohort-averaged parameters including Point A dose, ICRU rectum dose, and ICRU bladder dose for conventional planning, and clinical target volume (CTV) $\mathrm{D}_{90}$ and $\mathrm{D}_{2 \mathrm{cc}}$ for rectum, bladder, and sigmoid for volume-optimized planning, were reduced by $\leq 2.6 \%$ and often, by $\leq 2 \%$. The authors estimated that attenuation in the titanium applicator walls contributed $\sim 1.3 \%$ to these reductions.

Mikell et al. also studied conventional, CT-based tandem and ovoids HDR ${ }^{192}$ Ir treatment plans that incorporated stainless steel shielding in the ovoids, using 
BrachyVision v.10.0.26 in a retrospective study involving 24 patients [37]. Using the same dose parameters and heterogeneity factors employed in their earlier study [38] and again reporting $D_{\mathrm{w}, \mathrm{m}}$, the authors found reductions relative to TG-43 of up to $3.8 \%, 11.9 \%, 7.2 \%$, and $9.3 \%$ for Point $A$ dose, $D_{2 c c}$ rectum, $D_{2 c c}$ bladder, and $D_{2 c c}$ sigmoid, respectively. Reductions in doses to organs at risk were predominately associated with the applicator. Some inconsistencies in applicator properties modelled in the BrachyVision applicator library and obtained from radiographic measurements were noted, although these were deemed not to be of clinical concern.

Lastly, Hofbauer et al. recalculated HDR ${ }^{192}$ Ir treatment plans for nine cervix patients delivered with plastic tandem and ring applicators, for which applicator models were not available, using Acuros BV in BrachyVision v.10.0 [41]. Five of the nine patients were treated with a combined intracavitary/ interstitial technique. Based on reporting $D_{\mathrm{w}, \mathrm{m}^{\prime}}$ the authors observed only a very small dosimetric impact, with $\mathrm{D}_{90}$ and $\mathrm{V}_{100}$ for high-risk CTV reduced by $<0.5 \%$, and $\mathrm{D}_{2 \mathrm{cc}}$ and $\mathrm{D}_{0.1 \mathrm{cc}}$ for organs at risk reduced by $<2 \%$.

\section{Breast}

Zourari et al. compared TG-43, MC, and AcurosBV (reporting $D_{\mathrm{w}, \mathrm{m}}$ in BrachyVision v.8.8) HDR ${ }^{192}$ Ir dose calculations for a voxelized mathematical phantom resembling an interstitial breast brachytherapy patient, as well as for an actual patient [39]. For the phantom, which had a PTV volume of $7.25 \mathrm{~cm}^{3}$, PTV coverage was similar for all dose calculation methods. However, TG-43 increasingly overestimated dose relative to the other methods with increasing distance from the PTV (typically by $>5 \%$ at $2 \mathrm{~cm}$ distance) due to the combined effect of the lung heterogeneity and the phantom boundary. Furthermore, while perhaps not of clinical concern, TG-43 underestimated the dose within the volume of the flexible catheters by more than $25 \%$, likely as a result of the dose extrapolation algorithm employed by the TPS. For the patient, who had a PTV volume of $45.9 \mathrm{~cm}^{3}$, similar trends were observed. In particular, TG-43 overestimated dose to lung by up to $10 \%$, and dose to breast skin by up to $20 \%$. AcurosBV agreed with $\mathrm{MC}$ within $\pm 2 \%$, except for small volumes close to the source mostly inside the catheters.

For a cohort of 38 patients who received accelerated partial breast irradiation (APBI) via multi-catheter interstitial HDR ${ }^{192}$ Ir brachytherapy treatment, Zourari et al. compared ACE $\left(D_{m, m}\right)$ and TG-43 dosimetry using Oncentra Brachy v.4.4 [42]. An analysis of clinically relevant dose-volume parameters for PTV, breast, lung, heart, rib, and skin revealed statistically significant differences for all parameters, except for heart. However, PTV differences were relatively small $(<1 \%$ for coverage), with TG-43 overestimating only slightly. Larger differences for rib $\left(4 \%\right.$ for $\left.\mathrm{D}_{\max }\right)$, lung ( $5 \%$ for $\left.\mathrm{V}_{10 \mathrm{~Gy}}\right)$, and skin $\left(\sim 6 \%\right.$ for $\left.\mathrm{D}_{10 \mathrm{cc}}\right)$ were correlated with target location. The authors concluded that although the MBDCA appeared to improve dosimetric accuracy, differences from TG-43 did not warrant changing the dose prescription or the treatment protocol.

More recently, Ma et al. compared ACE $\left(D_{\mathrm{m}, \mathrm{m}}\right)$ and TG-43 dosimetry using Oncentra Brachy v.4.5 for an
APBI HDR ${ }^{192}$ Ir breast treatment delivered using 7-channels of a SAVI applicator [31]. They reported TG-43 PTV parameters $\mathrm{V}_{100}, \mathrm{D}_{90}$, and $\mathrm{D}_{50}$ to be $\sim 2 \%$ higher, $\mathrm{V}_{150}$ to be $5 \%$ higher, and $V_{200}$ to be $18 \%$ higher. All the ACE-calculated parameters agreed to $<1 \%$ with reference values calculated using MC. However, ACE disagreement with MC observed in the 3D dose distributions, both in nearsource high-dose regions and scatter-dominated lowdose ( $\leq 10 \%$ of the prescribed dose) regions, was deemed to deserve further evaluation.

Additional HDR ${ }^{192}$ Ir APBI work has been reported by Hofbauer et al. using AcurosBV (calculating $D_{\mathrm{w}, \mathrm{m}}$ ) for ten patients treated with flexible plastic catheters [41], and by Thrower et al. using ACE (calculating $D_{\mathrm{m}, \mathrm{m}}$ ) for 50 patients treated with a SAVI or Contura device [43]. These studies found dose differences for the CTV and OARs relative to TG-43 to be consistent with those of earlier investigations, insofar as direct comparisons were possible.

\section{Prostate}

Ma et al. also compared ACE $\left(D_{\mathrm{m}, \mathrm{m}}\right)$ and TG-43 dosimetry for a 17 -catheter HDR ${ }^{192} \mathrm{Ir}$ prostate implant [31]. PTV parameters $V_{100}, D_{90}$, and $D_{50}$ were found to be $<1.8 \%$ higher for TG-43, $\mathrm{V}_{150}$ was $13 \%$ higher, $\mathrm{V}_{200}$ was $48 \%$ higher, and rectum (air-filled to present a worst-case scenario) $\mathrm{D}_{2 \mathrm{cc}}$ was $12 \%$ higher. Relative to $\mathrm{MC}$ reference values, all ACE parameters were lower by no more than $1.3 \%$, with the exception of PTV $V_{150}$ (4\% lower) and $V_{200}$ (16\% lower). The underestimation of $\mathrm{V}_{200}$ is consistent with the observation made by the authors for the SAVI breast treatment that ACE overestimates dose in the nearsource region, considering that the $\mathrm{V}_{200}$ volume is more tightly bound to the catheters for the prostate implant than for the breast treatment. It was further noted by comparison to MC that ACE distinctly underestimated the low dose in pelvic bone, possibly because its scatter kernels are generated in water and the current implementation is unable to account for the different energy spectra of scattered photons in bone vs. water [9].

\section{Other}

Other HDR ${ }^{192}$ Ir treatment sites for which ACE or AcurosBV have been studied include esophagus [39], chest wall [31], lung [31], and head and neck [44]. Although clinical results for the hybrid Monte Carlo algorithm in the newer Radiance TPS have yet to be reported, a comparison of TG-43 and TG-186 dosimetry (both $D_{\mathrm{m}, \mathrm{m}}$ and $D_{\mathrm{w}, \mathrm{m}}$ were calculated using a public-domain MC code) for 7 breast irradiation patients treated using a low energy electronic brachytherapy source provides some insight into what might be expected [45]. The interested reader is referred to these publications for details.

\section{Work by the Authors}

Our center is conducting an experimental verification of ACE for a multi-channel vaginal applicator and, in collaboration with Elekta Brachytherapy, is involved in ongoing work examining the application of ACE to eye plaque treatments with ${ }^{125} \mathrm{I}$ sources. Selected findings 
from these works in progress are presented below. This material has been included solely to provide a glimpse of potential future developments for commercial MBDCAs; full accounts will be reported elsewhere.

\section{Multi-channel vaginal cylinder}

We compared the surface dose calculated by ACE to radiochromic film measurements for HDR ${ }^{192}$ Ir irradiation using a 35-mm diameter multi-channel vaginal cylinder (MCVC) (Elekta, Stockholm, SE, part \#110.761) with a vaginal tube (part \#101.002). The applicator has eight peripheral channels (Figure 1A) and a central channel provided by the vaginal tube, and is modeled in the applicator library of Oncentra Brachy v.4.5. Fixation of the applicator is achieved by attaching a perineal bar to two $5 \mathrm{~mm}$ deep grooves on the outside (Figure 1B). We routinely employ fixation for PDR treatments, but not for HDR treatments. For the latter, a condom is placed around the applicator prior to insertion into the patient.

Planning was performed using TG-43 calculations in Oncentra Brachy. A dose of 5 Gy was specified and geometrically optimized to 240 points placed uniformly on the applicator surface over a length of $70 \mathrm{~mm}$, using 20 dwell positions in the central channel. Dose was then recalculated with hACE and SACE for the same dwell times. For SACE, the applicator surface falls within the region of the calculation grid having $2 \mathrm{~mm}$ resolution [11]. For ACE calculations, the applicator was placed in a $30 \mathrm{~cm}^{3}$ virtual water box and the two grooves on the outside of the applicator were filled with air. Uncertainties for the TG-43 and ACE calculations were estimated to be $3.4 \%$ and $5 \%$, respectively $[13,16,46]$.

Measurements were made with Gafchromic EBT3 film (Ashland Specialty Ingredients, Wilmington, DE, USA) wrapped around the outside of the applicator and held in
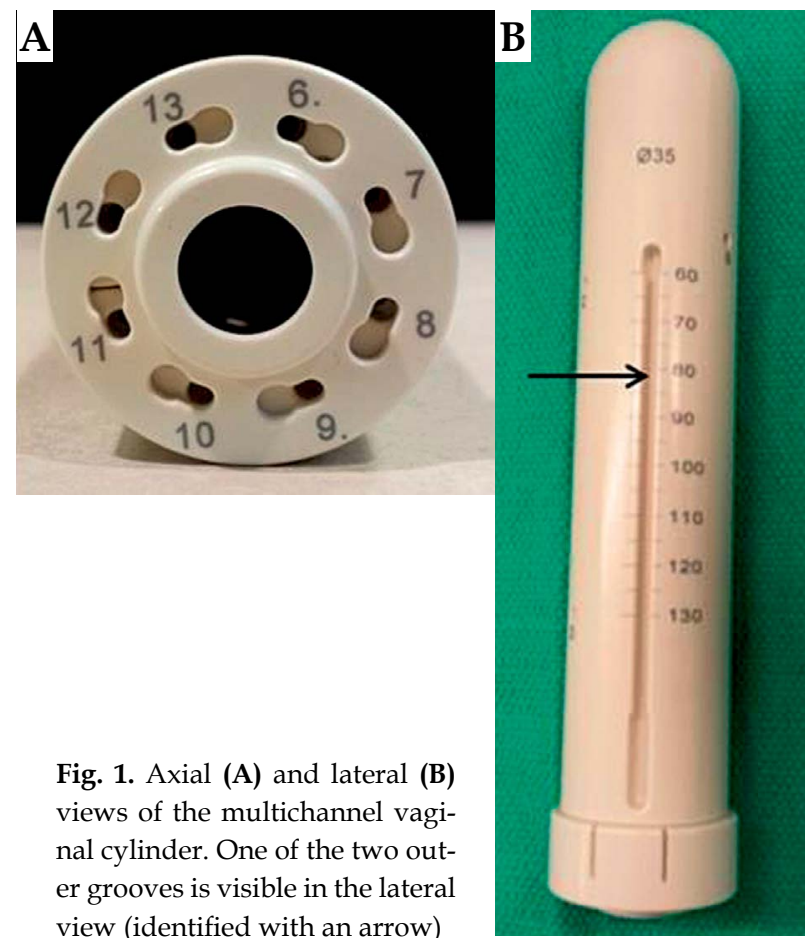

place with an acrylic sleeve. Irradiations were performed in a $30 \mathrm{~cm}^{3}$ water tank according to the TG-43-based plan for two scenarios: with water in the applicator grooves, achieved by placing the applicator in the tank prior to assembly (water-in-grooves set-up), or with air in the grooves, achieved by placing a condom over the assembled applicator, film and sleeve prior to placement in the tank (air-in-grooves set-up). The dose was delivered using a HDR ${ }^{192}$ Ir microSelectron ${ }^{\circledR}$ v.3 afterloader (Elekta, Stockholm, Sweden). Uncertainties in the film measured doses follow from the uncertainty analyses reported by ChiuTsao et al. [47] and Morrison et al. [48]. The dose uncertainty for an individual film pixel was $1-2 \%$.

The lengthwise-averaged dose at the surface of the applicator measured by film and calculated with ACE and TG-43 is plotted in Figure 2 as a function of azimuthal angle for the air-in-grooves set-up. The film measurements revealed a dose increase above the grooves of $11 \pm 4 \%$ $(k=1)$ of the mean surface dose, which was not seen for the water-in-grooves set-up. A $6 \pm 4 \%(k=1)$ increase in dose was observed for the hACE calculation, but no increase was seen for the sACE calculation. Apparently, the unadapted $2 \mathrm{~mm}$ grid size used for SACE at the applicator surface does not permit resolution of the applicator-air and air-water interfaces, sufficient for accurate dose calculation there. Implementation of an intelligent dose calculation grid for SACE that can adapt to the applicator surface could mitigate this issue. The film measurements also revealed a gradual variation in average measured dose around the applicator, seen as higher doses near 50 degrees and lower doses near 200 degrees. This is most likely caused by off-center positioning of the $0.9 \mathrm{~mm}$ diameter source in the $2.5 \mathrm{~mm}$ diameter central channel. Image-

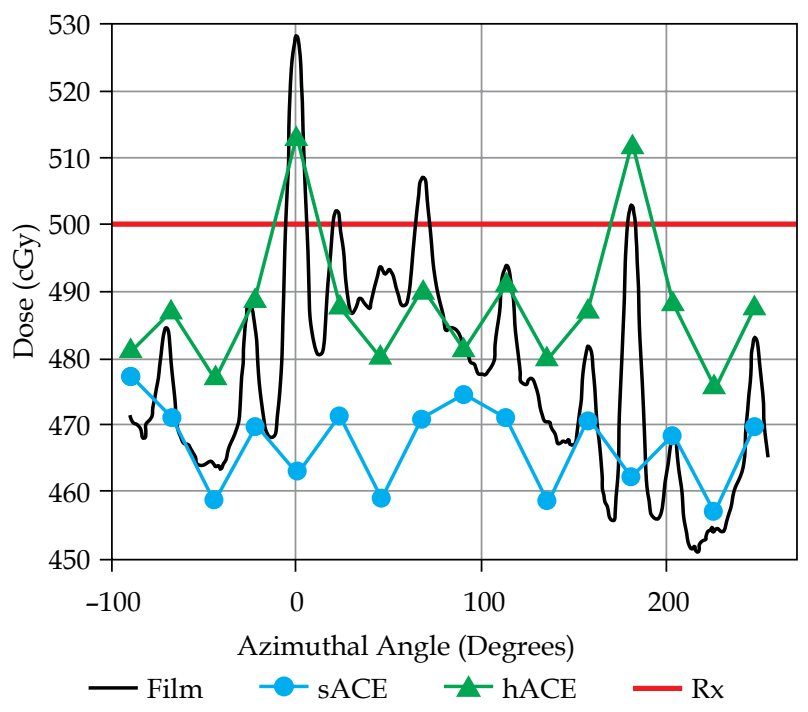

Fig. 2. Lengthwise-averaged dose on the surface of the MCVC applicator as a function of azimuthal angle for the air-in-grooves set-up with the central channel loaded. Angles $0^{\circ}$ and $180^{\circ}$ correspond to the outer grooves on the applicator. Dose was calculated using SACE and hACE for a single set of dwell times designed (using TG-43) to deliver 500 cGy (specified dose, $\mathrm{Rx}$ ) to points on the surface of the applicator. Dose was measured using radiochromic film 
based verification of source positions during treatment could enable this geometric uncertainty to be identified directly.

\section{COMS eye plaque}

A recommended dosimetry protocol for eye plaque therapy involves augmenting TG-43 calculations with TG-186 calculations that account for plaque materials, but assume all patient tissues are water equivalent, as TG- 43 calculations can overestimate the dose by $>20 \%$ in front of the plaque [35]. In the absence of a MBDCA for low-energy sources, this can be done using look-up tables of TG-186 doses along the plaque central axis (CAX) [35], or by applying correction factors $[49,50,51]$ to TG- 43 calculations as implemented in Plaque Simulator software (Eye Physics LLC, Los Alamitos, CA, USA).

In collaboration with Elekta Brachytherapy, we introduced a $12 \mathrm{~mm}$ COMS plaque (see Figure 3 inset) into Oncentra Brachy v.4.5.2 by creating 3D CAD models of the plaque backing (Modulay) and Silastic insert, and adding these to the applicator library. Material compositions and densities reported in TG-129 were used for the applicator model [35]. A Nucletron selectSeed (Elekta Brachytherapy p.n.130.002) ${ }^{125}$ I source model for ACE was created solely for research purposes from primary-scatter separated kernel data generated by the CLRP group [52,53]. Treatment plans were made for three source/plaque configurations located at the center of a $30 \mathrm{~cm}^{3}$ water box: 1) a single seed alone; 2 ) a single seed in the plaque central slot (SS-COMS); 3) a fully loaded plaque (FL-COMS).

For the single seed in water, a dose of 5 Gy was specified at $1 \mathrm{~cm}$ from the seed center on the transverse axis and the corresponding dwell time determined using TG-43 calculations. The dose distribution was then recalculated with hACE using a dose calculation grid size of $0.5 \mathrm{~mm}^{3}$, which was used for all calculations reported here. For the SS-COMS plan, a dose of 5 Gy was specified at $7.4 \mathrm{~mm}$ from the center of the seed on the plaque CAX (5 $\mathrm{mm}$ from the inner sclera of a standard-sized spherical eye with $12.3 \mathrm{~mm}$ radius and $1 \mathrm{~mm}$ scleral thickness), the dwell time was determined using TG-43, and dose was recalculated. The same procedure was followed for the FL-COMS plan, but with 70 Gy specified at $7.4 \mathrm{~mm}$ from the central seed. For all three configurations, dose data were exported from a $60 \mathrm{~mm}^{3}$ region of interest (ROI) centered on the central seed and having $0.5 \mathrm{~mm}^{3}$ voxels. For comparison, reference dose data were created using Monte Carlo simulations with MCNP6 (v.1) [54]. MCNP6 simulations were performed with $5 \times 10^{9}$ starting particles per seed in Mode P and dose was scored using the F6 and ${ }^{*}$ FMESH tallies. The seed air kerma strength was determined in a separate simulation and scaled to match that used in Oncentra Brachy.

For the single seed in water, average dose differences within the ROI between ACE and TG-43, TG-43 and MCNP6, and ACE and MCNP6 were $0.78 \pm 2.3 \%, 3.7 \pm$ $3.0 \%$, and $4.4 \pm 2.1 \%$, respectively (mean \pm standard deviation). The largest differences were noted for the TG-43 data near the seed end-welds. The ACE data within Oncentra Brachy exhibits differences from more recent TG-43

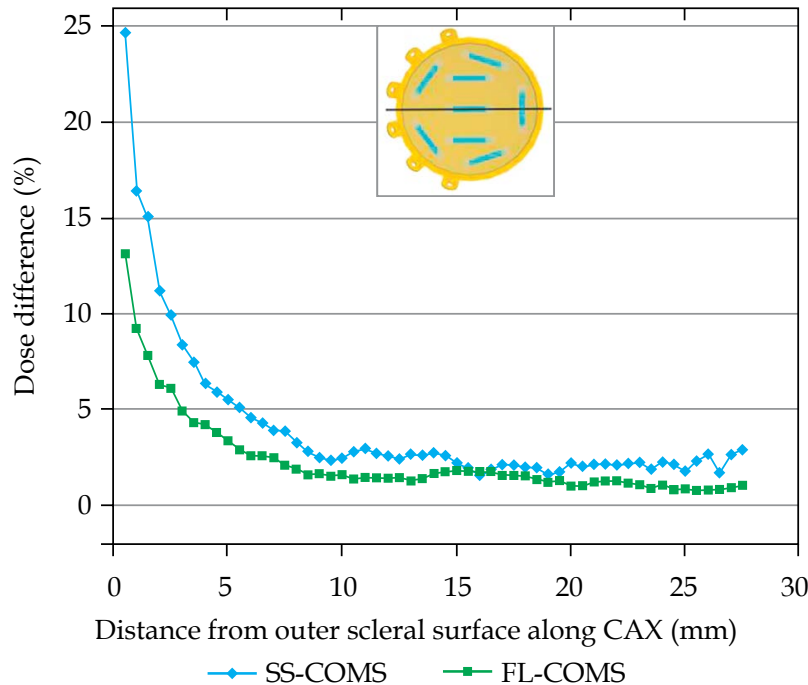

Fig. 3. Percentage dose differences along the plaque $C A X$ between ACE and MCNP6 for the SS-COMS and FL-COMS plans $\left(\%\right.$ diff $=\left(\right.$ Dose $_{\mathrm{ACE}}-$ Dose $\left._{\mathrm{MCNP}}\right) /$ Dose $\left.\left._{\mathrm{MCNP6}}{ }^{*} 100 \%\right)\right)$. Inset: front view of the $12 \mathrm{~mm}$ COMS plaque showing the eight seed slots and the orientation of the central dose calculation plane

Monte Carlo data for selectSeed (obtained independently by our group and the CLRP group, which agreed on average within 1\%), which is likely the cause of some of the differences seen between TG-43/ACE and MCNP6.

For the plaque plans, ACE was able to predict the decrease in dose relative to TG-43 along the plaque CAX; dose differences between ACE and MCNP6 averaged over a length of $27 \mathrm{~mm}$ were $4.1 \pm 4.2 \%$ for SS-COMS, and $2.3 \pm 2.3 \%$ for FL-COMS. The percent differences along the CAX between ACE and MCNP6 for both plans are shown in Figure 3. The poorer agreement observed at distances $<5 \mathrm{~mm}$ from the inner sclera is the subject of ongoing investigation.

Percent dose differences between ACE and MCNP6 in the central plane of the plaque are shown in Figure 4 for the SS-COMS and FL-COMS plans. The largest clinically relevant differences are seen in the plaque penumbrae, and likely arise from simplifications made in radiation source modelling and angular discretization of photon transport in ACE [9]. The combined effect can be seen as single high and low percentage difference streaks near the plaque lip for SS-COMS, and multiple streaks (due to multiple seeds) for FL-COMS. Within the standard eye model volume directly in front of the plaque, average percent dose differences are $1.5 \pm 6.7 \%$ and 2.1 $\pm 6.8 \%$ for SS-COMS and FL-COMS, respectively. Thus, overall agreement is good within this volume, however due to streak artifacts in the penumbrae, further analysis is required to determine how accurately ACE can calculate dose throughout. This is important because tissues at risk (optic nerve, macula, fovea) might be located near the plaque edge, depending on the position of the plaque on the eye. As noted for the MCVC results presented in the preceding section, an applicator-based adaptive dose calculation grid could merit investigation. 

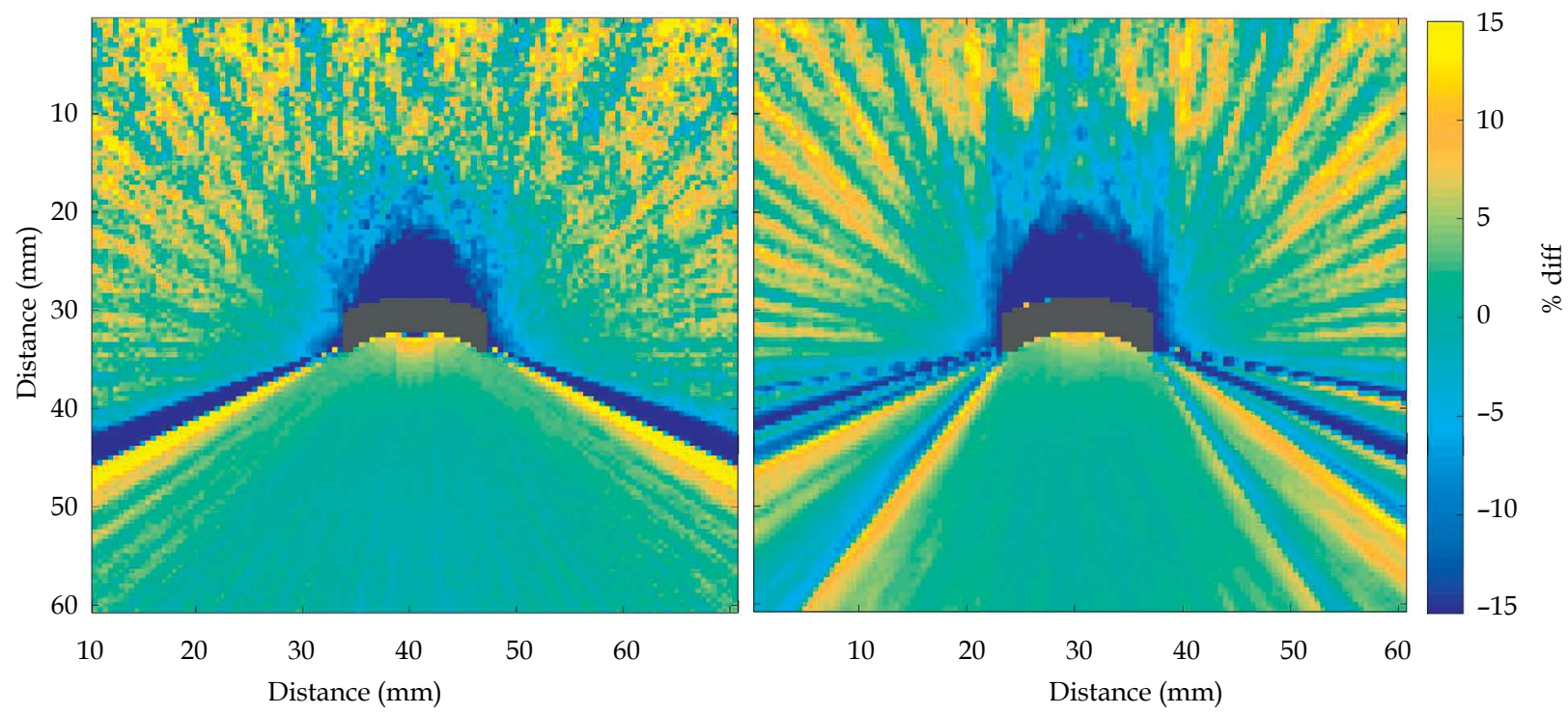

Fig. 4. Percentage dose differences between ACE and MCNP6 in the central plane (see Figure 3 inset) of a $12 \mathrm{~mm}$ COMS plaque for the SS-COMS plan (left), and the FL-COMS plan (right) $\left(\%\right.$ diff $=\left(\right.$ Dose $_{\mathrm{ACE}}-$ Dose $\left._{\mathrm{MCNP}}\right) /$ Dose $\left.\left._{\mathrm{MCNP}}{ }^{*} 100 \%\right)\right)$; pixels containing plaque materials have been greyed out

\section{Summary and outlook}

The TG-43 formalism provides a standardized approach to brachytherapy dose calculation based on the superposition of single-source dosimetry in water medium that is simple to use, but lacks accuracy to varying degrees in many clinical circumstances. Emergent commercial MBDCAs whose correct application is addressed in TG-186 are ideally capable of both closely reproducing TG-43 dosimetry in water and closely approximating Monte Carlo reference dosimetry in any clinical situation. This enhanced capability comes at the additional cost of adequately modelling the geometry and material composition of the patient in treatment position (as determined from imaging), and the radiation source and treatment applicator (vendor-supplied) for dose calculation purposes. Evaluation of a MBDCA's adequacy by investigators and clinical users alike needs to be done on a source, applicator, and treatment site specific basis. It is well underway for HDR ${ }^{192}$ Ir sources and the IntraBeam XRS-4 source, has recently begun for ${ }^{125}$ I seeds, and will follow the implementation timetables of TPS vendors for other brachytherapy sources and applicators. Accordingly, the transition from TG-43 to TG-186 based dosimetry will necessarily occur over an extended period of time.

The enhanced dose calculation accuracy that MBDCAs can provide is contingent on the geometric accuracy of the treatment model used for calculation. Uncertainties in 1) source positions and orientations; 2) applicator position, orientation, and description; 3) patient anatomical structure segmentation, and 4) patient anatomy variations over the course of the irradiation period can potentially reduce the accuracy of calculated dose estimates. Therefore, geometric uncertainties need to be well understood and, if necessary, mitigated in order to take full clinical advantage of the potential of MBDCAs. This observation is borne out in several of the selected experiences presented here in the clinical experience section. Coincidentally, the Physics
Workshop on Treatment Verification held in conjunction with the 2016 World Congress of Brachytherapy meeting [55] included reports on several different technologies in various stages of commercial development that can help minimize geometric uncertainties in the treatment model. Likewise, vendor-supplied applicator material compositions and user-selected clinical mappings of patient images to tissue types, bear careful scrutiny prior to clinical use.

Within the past decade, the international study on MRI-guided brachytherapy in locally advanced cervical cancer and related retroEMBRACE study have clearly demonstrated that image-guided adaptive brachytherapy can substantially improve disease outcome and reduce treatment-related morbidity [56]. Could the use of MBDCAs serve to facilitate similar improvements in treatment techniques and outcomes at other treatment sites, for which TG-43 dosimetry is demonstrably inaccurate? Despite new challenges associated with MBDCA familiarization, commissioning, clinical implementation, and quality assurance, the potential for such improvements definitely exists, especially for low-energy sources. The extent to which these can be identified and realized will depend in large part on how well practical challenges are met and solutions shared by commercial MBDCA vendors, investigators, and users alike.

\section{Disclosure}

Authors report no conflict of interest.

\section{References}

1. Nath R, Anderson L, Luxton G et al. Dosimetry of interstitial brachytherapy sources: Recommendations of the AAPM Radiation Therapy Committee Task Group No. 43. Med Phys 1995; 22: 209-234.

2. Rivard M, Coursey B, DeWerd L et al. Update of AAPM Task Group No. 43 report: A revised AAPM protocol for brachytherapy dose calculations. Med Phys 2004; 31: 633-674. 
3. Pérez-Calatayud J, Ballester J, Das RK et al. Dose calculation for photon-emitting brachytherapy sources with average energy higher than $50 \mathrm{keV}$ : Report of the AAPM and ESTRO. Med Phys 2012; 39: 2904-2929.

4. Rusch T, Rivard M. Application of the TG-43 Dosimetry Protocol to Electronic Brachytherapy Sources. Presented at Joint Brachytherapy Meeting of GEC/ESTRO/ABS, 2004. http:// www.xoftinc.com/treating_physics.html. Accessed: 14 June 2016.

5. Rivard MJ, Venselaar J, Beaulieu L. The evolution of brachytherapy treatment planning. Med Phys 2009; 36: 2136-2153.

6. Chibani O, Williamson JF, Todor D. Dosimetric effects of seed anisotropy and interseed attenuation for ${ }^{103} \mathrm{Pd}$ and ${ }^{125} \mathrm{I}$ prostate implants. Med Phys 2005; 32: 2557-2566.

7. Beaulieu L, Carlsson Tedgren A, Carrier J et al. Report of the Task Group 186 on model-based dose calculation methods in brachytherapy beyond the TG-43 formalism: Current status and recommendations for clinical implementation. Med Phys 2012; 39: 6208-6236.

8. Varian Medical Systems. Acuros BV Algorithm Reference Guide. Document ID: B504878R01A, Revision A, August 2013.

9. Ahnesjö A, van Veelen B, Carlsson Tedgren $\AA$. Collapsed cone dose calculations for heterogeneous tissues in brachytherapy using primary and scatter separation source data. Comput Methods Programs Biomed 2017; 139: 17-29.

10. Soluciones Globales Internet S.A.U. Document ID: GMV-RADIANCE-SUM-EN, version 2.5. Assessed: 19 October 2016

11. ACE: Advanced Collapsed Cone Engine. White paper Elekta Brachytherapy, 2014. http://blog.elekta.com/blog/ 2015/04/new-elekta-brachytherapy-white-papers/ Accessed 7 June 2016.

12. Radiance. White paper, GMV SA, 2015. http://www.gmv com/export/sites/gmv/DocumentosPDF/radiance/radiance-white-paper-en_2015.pdf. Accessed 10 November 2016.

13. Papagiannis P, Pantelis E, Karaiskos P. Current state of the art brachytherapy treatment planning dosimetry algorithms. Br J Radiol 2014; 87: 1-20.

14. Russell KR, Carlsson Tedgren $\AA$, Ahnesjö A. Brachytherapy source characterization for improved dose calculations using primary and scatter dose separation. Med Phys 2005; 32: 2739-2752

15. Gifford KA, Horton Jr JL, Wareing TA et al. Comparison of a finite-element multigroup discrete-ordinates code with Monte Carlo for radiotherapy calculations. Phys Med Biol 2006; 51: 2253-2265.

16. Ballester F, Carlsson Tedgren $\AA$, Granero D et al. A generic high-dose rate ${ }^{192}$ Ir brachytherapy source for evaluation of model-based dose calculations beyond the TG-43 formalism. Med Phys 2015; 42: 3048-3061.

17. Carlsson Tedgren $\AA$, Alm Carlsson G. Specification of absorbed dose to water using model based dose calculation algorithms for treatment planning in brachytherapy. Phys Med Biol 2013; 58: 2561-2579.

18. Enger SA, Ahnesjö A, Verhaegen F et al. Dose to tissue medium or water cavities as surrogate for the dose to cell nuclei at brachytherapy photon energies. Phys Med Biol 2012; 57: 4489-4500.

19. Thomson RM, Carlsson Tedgren $\AA$, Williamson JF. On the biological basis for competing macroscopic dose descriptors for kilovoltage dosimetry: cellular dosimetry for brachytherapy and diagnostic radiology. Phys Med Biol 2013; 58: 1123-1150.

20. Tedgren ÅC, Plamondon M, Beaulieu L. The collapsed cone algorithm for ${ }^{192} \mathrm{Ir}$ dosimetry using phantom-size adaptive multiple-scatter point kernels. Med Phys 2015; 13: 5313-5323.

21. Tedgren $\AA \mathrm{C}$, Ahnesjö A. Accounting for high $\mathrm{Z}$ shields in brachytherapy using collapsed cone superposition for scatter dose calculation. Med Phys 2003; 30: 2206-2217.
22. Vidal M, Guerra P, Herranz E et al. Realistic on-the-fly dose calculation for low energy X-rays Intra-Operative Radiation Therapy. Radiother Oncol 2014; 110 (Suppl 1): S103-104.

23. Vidal M, Ibáñez P, González J et al. Hybrid Monte Carlo dose algorithm for low energy x-rays intra-operative radiation therapy. Radiother Oncol 2014; 111 (Suppl 1): S106-S107.

24. Kutcher G, Coia L, Gillin M et al. Comprehensive QA for radiation oncology: Report of AAPM Radiation Therapy Committee Task Group 40. Med Phys 1994; 21: 581-618.

25. Nath R, Anderson L, Meli J et al. Code of practice for brachytherapy physics: Report of the AAPM Radiation Therapy Committee Task Group No. 56. Med Phys 1997; 24: 1557-1598.

26. Venselaar J, Pérez-Calatayud J [eds.]. A practical guide to quality control of brachytherapy equipment. European Guidelines for Quality Assurance in Radiotherapy Booklet No. 8, European Society for Radiotherapy \& Oncology, Brussels 2004.

27. Kubo D, Glasgow G, Pethel T et al. High dose-rate brachytherapy treatment delivery: Report of the AAPM Radiation Therapy Committee Task Group No. 59. Med Phys 1998; 25: 375-403.

28. Beaulieu L, Ballester F, Carlson-Tedgren A et al. Implementation and validation of an end-to-end commissioning process for model-based dose calculation algorithms in brachytherapy. Brachytherapy 2016; 15 (Suppl 1): S172.

29. Working group on model-based dose calculation algorithms in brachytherapy. http://www.aapm.org/org/structure/ default.asp?committee_code=WGDCAB. Accessed 16 August 2016.

30. Peppa V, Pantelis E, Pappas E et al. A user-oriented procedure for the commissioning and quality assurance testing of treatment planning system dosimetry in high-dose-rate brachytherapy. Brachytherapy 2016; 15: 252-262.

31. Ma Y, Lacroix F, Lavallee $\mathrm{M}$ et al. Validation of the Oncentra Brachy Advanced Collapsed-cone Engine for a commercial ${ }^{192}$ Ir source using heterogeneous geometries. Brachytherapy 2015; 14: 939-952.

32. Fonseca G, Reniers B, Landry G et al. A medical image-based graphical platform - features, applications and relevance for brachytherapy. Brachytherapy 2014; 13: 632-639.

33. Afsharpour H, Landry G, D'Amours M et al. ALGEBRA: ALgorithm for the heterogeneous dosimetry based on GEANT4 for BRAchytherapy. Phys Med Biol 2012; 57: 3273-3280.

34. Petrokokkinos L, Zourari K, Pantelis E et al. Dosimetric accuracy of a deterministic radiation transport based ${ }^{192} \mathrm{Ir}$ brachytherapy treatment planning system. Part II: Monte Carlo and experimental verification of a multiple source dwell position plan employing a shielded applicator. Med Phys 2011; 38: 1981-1992.

35. Chiu-Tsao S, Astrahan M, Finger P et al. Dosimetry of ${ }^{125} \mathrm{I}$ and ${ }^{103} \mathrm{Pd}$ COMS eye plaques for intraocular tumors: Report of Task Group 129 by the AAPM and ABS. Med Phys 2012; 39: 6161-6184.

36. Thomson R, Furutani K, Kaulich $\mathrm{T}$ et al. Recommendations on medical physics practices for ocular plaque brachytherapy: Report of Task Group 221 by the AAPM. Med Phys 2016 [In preparation].

37. Mikell J, Klopp A, Price $M$ et al. Commissioning of a gridbased Boltzmann solver for cervical cancer brachytherapy treatment planning with shielded colpostats. Brachytherapy 2013; 12: 645-653.

38. Mikell J, Klopp A, Gonzalez G et al. Impact of heterogeneity-based dose calculation using a deterministic grid-based Boltzmann equation solver for intracavitary brachytherapy. Int J Radiat Oncol Biol Phys 2012; 83: e417-e422.

39. Zourari K, Pantelis E, Moutsatsos A et al. Dosimetric accuracy of a deterministic radiation transport based ${ }^{192}$ Ir brachythera- 
py treatment planning system. Part III. Comparison to Monte Carlo simulation in voxelized anatomical computational models. Med Phys 2013; 40: 011712:1-9.

40. Hyer D, Sheybani A, Jacobson G et al. The dosimetric impact of heterogeneity corrections in high-dose-rate ${ }^{192} \mathrm{Ir}$ brachytherapy for cervical cancer: Investigation of both conventional Point-A and volume-optimized plans. Brachytherapy 2012; 11: 515-520.

41. Hofbauer J, Kirisits C, Resch A et al. Impact of heterogeneity-corrected dose calculation using a grid-based Boltzmann solver on breast and cervix cancer brachytherapy. J Contemp Brachytherapy 2016; 8: 143-149.

42. Zourari K, Major T, Herein A et al. A retrospective dosimetric comparison of TG43 and a commercially available MBDCA for an APBI brachytherapy patient cohort. Phys Med 2015; 31: 669-676.

43. Thrower SL, Shaitelman SF, Bloom E et al. Comparison of dose distributions with TG- 43 and collapsed cone convolution algorithms applied to accelerated partial breast irradiation plans. Int J Radiat Oncol Biol Phys 2016; 95: 1520-1526.

44. Siebert F-A, Wolf S, Kóvacs G. Head and neck ${ }^{192} \mathrm{Ir}$ brachytherapy dosimetry using a grid-based Boltzmann solver. J Contemp Brachytherapy 2013; 5: 232-235.

45. White S, Landry G, Fonseca G et al. Comparison of TG-43 and TG-186 in breast irradiation using a low energy electronic brachytherapy source. Med Phys 2014; 41: 1-12.

46. DeWerd LA, Ibbott GS, Meigooni AS et al. A dosimetric uncertainty analysis for photon-emitting brachytherapy sources: report of AAPM Task Group No. 138 and GEC-ESTRO. Med Phys 2011; 38: 782-801.

47. Chiu-Tsao S-T, Medich D, Munro J III. The use of new Gafchromic EBT film for ${ }^{125} \mathrm{I}$ seed dosimetry in Solid Water phantom. Med Phys 2008; 35: 3787-3799.

48. Morrison H, Menon G, Sloboda RS. Radiochromic film calibration for low-energy seed brachytherapy dose measurement. Med Phys 2014; 41: 072101:1-11.

49. Thomson R, Taylor R, Rogers D. Monte Carlo dosimetry for 125 I and 103 Pd eye plaque brachytherapy. Med Phys 2008; 35: 5530-5543.

50. Luxton G, Astrahan M, Petrovich Z. Backscatter measurements from a single seed of 125 I for ophthalmic plaque dosimetry. Med Phys 1988; 15: 397-400.

51. Astrahan M. Improved treatment planning for COMS eye plaques. Int J Radiat Oncol Biol Phys 2005; 61: 1227-1242.

52. Taylor R, Rogers D. An EGSnrc Monte Carlo-calculated database of TG-43 parameters. Med Phys 2008; 35: 4228-4241.

53. Karaiskos P, Papgiannis P, Sakelliou L. Monte Carlo dosimetry of the selectSeed ${ }^{125}$ I interstitial brachytherapy seed. Med Phys 2001; 28: 1753-1760.

54. Goorley T, James M, Booth T et al. Initial MCNP6 Release Overview. Nucl Technol 2012; 180: 298-315.

55. World Congress of Brachytherapy 2016, San Francisco CA, June 27-29, 2016. Agenda at https://www.americanbrachytherapy.org/meetings/annual2016/agenda/. Accessed: $30 \mathrm{Au}-$ gust 2016.

56. Tanderup K, Lindegaard J, Kirisits $C$ et al. Image Guided Adaptive Brachytherapy in cervix cancer: A new paradigm changing clinical practice and outcome. Radiother Oncol 2016; 120: 365-369. 